\title{
D029
}

\section{Migration without Velocity Model in Curvelet Domain}

\author{
A. Bona* (Curtin University of Technology) \& M. AlMaghlouth (Saudi \\ Aramco)
}

\section{SUMMARY}

We present a formulation of velocity-less time migration in curvelet domain. In particular, we first decompose pre-stack gathers to curvelets, which unlike wavelets also contain directional information. Then, we use this directional information to perform migration that does not require any velocity model. This migration is performed directly with the curvelet coefficients. The resulting image is reconstructed by applying the inverse curvelet transform. The process is illustrated on synthetic data and compared to Kirchhoff migration. 


\section{Introduction}

It is possible to find the velocity and the reflector below a constant velocity layer by using the traveltimes of the reflected rays and their horizontal slownesses, which are also called local event slopes. The idea of using horizontal slownesses to obtain the migration velocity as well as the migrated location goes back to Puzyrev, Riznichenko and Rudnev in 1945. Goldin (1986) was probably the first to publish the approach in English literature. Sword (1987) presents this constant velocity depth migration alongside his tomography approach. Migration proposed by Fomel (2007) is equivalent to these migrations. The idea of using horizontal slowness was also used by Kleyn (1977) for zero-offset migration, and byOttolini (1983) for migration of horizontal reflectors. Cooke et al. (2009) present another point of view on this velocityless migration, where they use the resulting migration velocity to perform multiples suppression. All of these formulations require the horizontal slownesses in two domains: some in common-offset and common-midpoint (e.g. (Fomel, 2007)), and some in common-shot and common-receiver domains (e.g. Cooke et al. (2009)). There are many methods of finding these horizontal slownesses and only few of these methods can find multiple values of slownesses at a point corresponding to multipathing. One of such methods is to compress the data using curvelets (Candès et al., 2006). Once the data is transformed into curvelet transform, it is possible to perform migration directly in this domain. (Douma and de Hoop, 2007) used similar approach for time migration with known velocity model. Herein, we will apply such approach to perform the time migration without a velocity model directly in the curvelet domain.

\section{Theory}

We begin this section with a brief introduction to curvelets, which were introduced by Candès and Donoho (2000). Herein, we give an abridged version of introduction to curvelets from Douma and de Hoop (2007) and (Candès, 2003), which are probably the most accessible references to geophysicists on the subject and contain many relevant references.

Curvelets form a tight frame of square-integrable functions in a real plane. This means that we express such functions using curvelets $c_{\mu}$ as

$$
f=\sum\left\langle f, c_{\mu}\right\rangle c_{\mu}
$$

where the scalar product is

$$
\left\langle f, c_{\mu}\right\rangle=\iint f(x, y) c_{\mu}(\bar{x}, y) d x d y
$$

and the following holds

$$
\iint f(x, y) f(\bar{x}, y) d x d y=\sum\left|\left\langle f, c_{\mu}\right\rangle\right|^{2} .
$$

Roughly speaking, curvelets can be obtained by applying parabolic dilatations, rotations and translations to a specific function $c$; they are indexed by a scale parameter $j$, orientation $i$ and location $\left(m_{1}, m_{2}\right)$ :

$$
c_{i j m}(x, y)=j^{-3 / 4} c\left(D_{j} R_{i}\left(x-m_{1}, y-m_{2}\right)\right),
$$

where

$$
D_{j}=\left(\begin{array}{cc}
1 / j & 0 \\
0 & 1 / \sqrt{j}
\end{array}\right)
$$

is a parabolic scaling matrix and $R_{i}$ is a rotation matrix controlled by index $i$. Figure 1 (left) from Candès (2003) illustrates several curvelets at different scales, rotations and translations. For digital curvelets one needs to consider discrete scaling, rotations and translations. Such discretisation of scaling and rotation can be done in the frequency domain by considering coronae illustrated in Figure 1 (middle). Discretisation of the translations in spatial domain is shown in Figure 1 (right). Decomposition of a seismic gather into curvelets provides not only a possibility to compress the data, but also provides information about the frequency content and orientation of the events. This orientation information can be utilised for time migration without any velocity model, as we discuss in the next paragraph. 

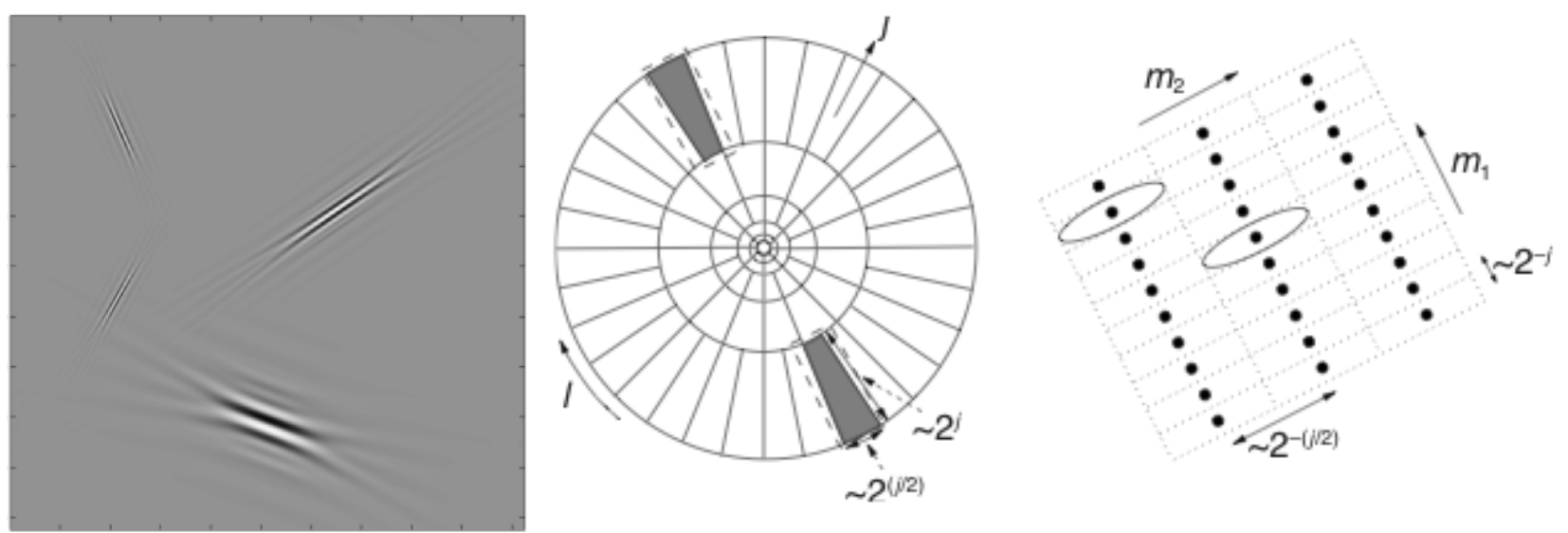

Figure 1 Example of four curvelets at different scales, rotations and translations (left). Taken from Candès (2003). Illustration of curvelet discretisation in frequency domain (middle) and in the spatial domain (right). The Fourier image of the particular curvelet is supported in the shaded area in the middle picture. Taken from Douma and de Hoop (2007).

To summarize the method of time migration using horizontal slownesses, we follow Cooke et al. (2009). The double square-root equation

$$
t=\sqrt{t_{0}^{2}+\frac{\left(x_{r}-x_{m}\right)^{2}}{v^{2}}}+\sqrt{t_{0}^{2}+\frac{\left(x_{s}-x_{m}\right)^{2}}{v^{2}}}
$$

describes the travel time of seismic reflections and diffractions in a homogeneous medium. In this equation, $t$ is travel time, $v$ is velocity, $t_{0}$ is vertical travel time to the reflector/ diffractor and $x_{m}, x_{s}$, and $x_{r}$ are the horizontal coordinates of the reflection point, source location, and receiver location. For all $(x, t)$ points in a shot or receiver gather, $t, x_{s}$, and $x_{r}$ are known while $v, t_{0}$ (or equivalently depth) and $x_{m}$ are unknowns. We will solve for these unknowns below.

We denote the partial derivative of equation 1 with respect to $x_{r}$ by $p_{s}$ (this is the $p$-value measured in a common-shot gather):

$$
p_{s}(x, t)=\frac{\partial t}{\partial x_{r}}=\frac{x_{r}-x_{m}}{v^{2} \sqrt{t_{0}^{2}+\left(x_{r}-x_{m}\right)^{2} / v^{2}}},
$$

and the partial derivative of equation 1 with respect to $x_{s}$ is defined by $p_{r}$ (this is $p$ measured in a common-receiver gather):

$$
p_{r}(x, t)=\frac{\partial t}{\partial x_{s}}=\frac{x_{s}-x_{m}}{v^{2} \sqrt{t_{0}^{2}+\left(x_{s}-x_{m}\right)^{2} / v^{2}}} .
$$

As we discuss later, $p_{s}$ and $p_{r}$ can be measured for every sample in shot and receiver gathers respectively. Once $p_{s}$ and $p_{r}$ are known, equations 1,2, and 3 represent a system of three equations with three unknowns $-x_{m}, v$, and $t_{0}$. The solution of these equations for the unknowns is by equations

$$
\begin{gathered}
x_{m}=x_{s}-d p_{s} \frac{t-d p_{r}}{t\left(p_{r}-p_{s}\right)+2 d p_{s} p_{r}}, \\
v^{2}=\frac{x_{s}-x_{m}}{t p_{s}}+\frac{x_{r}-x_{m}}{t p_{r}},
\end{gathered}
$$




$$
t_{0}=\frac{\left|x_{s}-x_{m}\right|}{v} \frac{\sqrt{1 / v^{2}-p_{s}^{2}}}{\left|p_{s}\right|} .
$$

where $d=x_{r}-x_{s}$ is the signed source-receiver offset.

If we decompose the shot and receiver gathers into curvelets, we can estimate the local slopes from the orientation of the individual curvelets, as shown in Figure 3. These curvelets can be than migrated using the migration equations 4,5 and 6 , where we use curvelets of the same scale index for both shot and receiver domain. The migrated curvelet is located according to the migration equations 4 and 6 with the same scale index as the input curvelets and with the orientation given by the equation

$$
\frac{1}{2}\left(\arccos \left(v p_{s}\right)+\arccos \left(v p_{r}\right)\right),
$$

where $p_{s}$ is the horizontal slowness in the shot gather and $p_{r}$ is the horizontal slowness in the receiver domain. The value of the coefficient of the migrated curvelet is the average of the coefficients from the decomposition of the shot and receiver gathers corresponding to the curvelets used for the migration. In the next section we exemplify the proposed method of migration in the curvelet domain.

\section{Example}

Herein, we demonstrate effectiveness of the above described migration using curvelet decomposition of synthetic data. Figure 2 shows the velocity model that we use to generate the data.

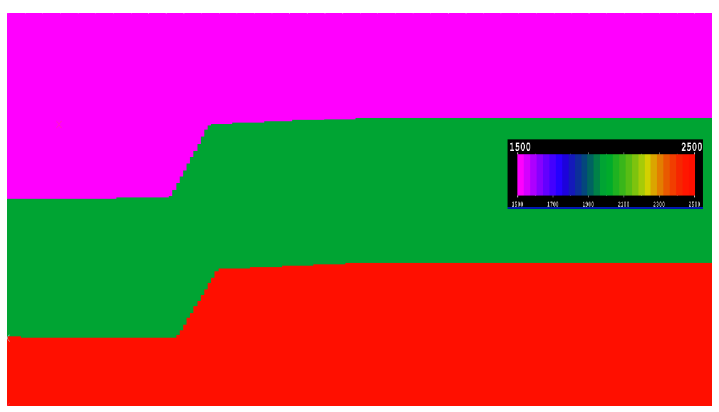

Figure 2 Velocity model used to generate the synthetic data.

After decomposition of shot and receiver gathers into curvelets, we perform compression to consider only the strongest events. In this particular case we use $0.2 \%$ of the curvelet coefficients for the migration. Figure 3 (left) shows the compressed reconstructed shot gather using only the considered curvelets. The information about the local slopes obtained from the curvelet coefficient is illustrated by the lines in Figure 3 (right).
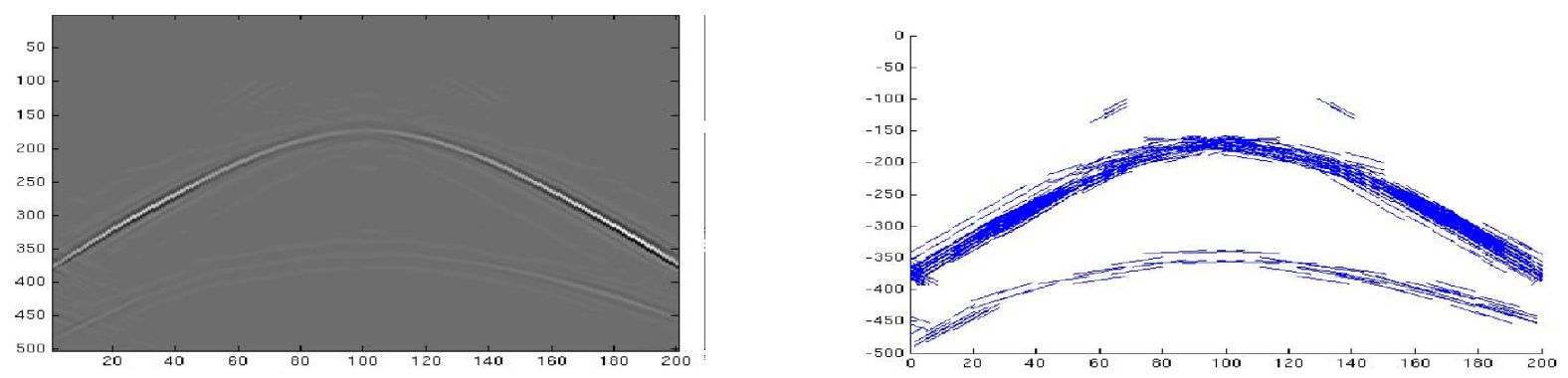

Figure 3 Shot gather compressed using $0.2 \%$ of the curvelet coefficients (left) and corresponding tangents (right). 
The result of the migration process, i.e. applying equations 4,5 and 6 to curvelet coefficients, is shown in Figure 4 (left). Effectiveness of the proposed technique can be judged by comparing this image with the image obtained by Kirchhoff migration with the true velocity model shown in Figure 4 (right).
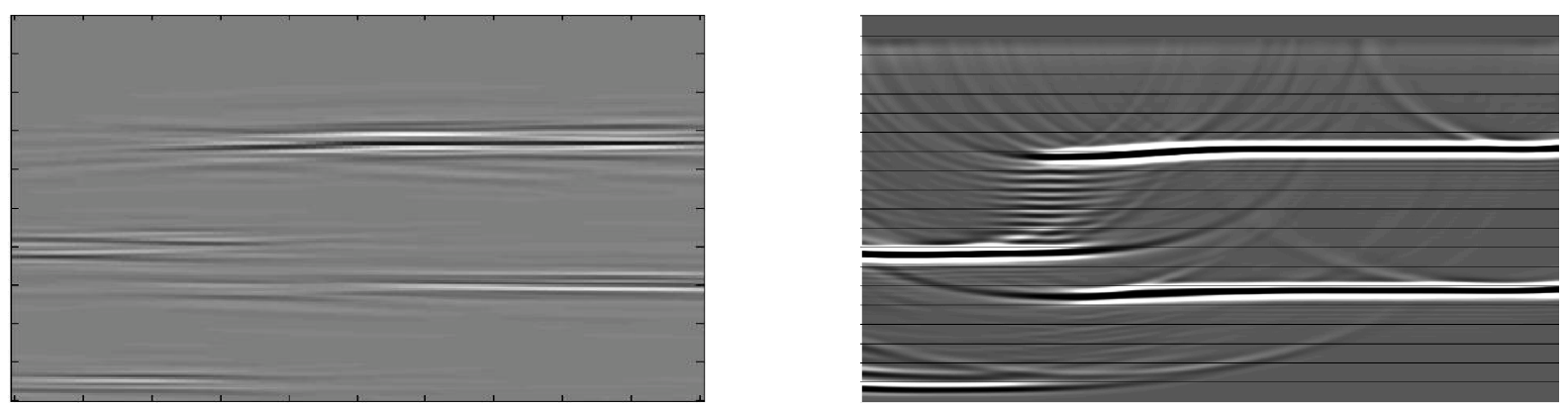

Figure 4

\section{Conclusions}

We have reformulated the time migration that uses local slopes to calculate the migration velocity in the curvelet domain. This reformulation has several advantages. First, the curvelet decomposition provides intrinsically information about the local slopes. Second, this approach allows finding local slopes at points of multipathing. Third, the algorithm works directly with the compressed data coefficients. Four, after the initial data compression, the algorithm is very fast. Also, as illustrated by comparison to the Kirchhoff migration - with known velocity model - the approach produces cleaner images: there are no migration smearing artifacts.

\section{References}

Candès, E.J. [2003] What is a curvelet? Notices of the AMS, 50(11).

Candès, E.J., Demanet, L., Donoho, D. and Ying, L. [2006] Fast discrete curvelet transforms. SIAM multiscale modeling and simulation, 5, 861-899.

Candès, E.J. and Donoho, L. [2000] Curvelets - a surprisingly effective nonadaptive representation for objects with edges. Curves and surface fitting: Saint-Malo 1999, Vanderbilt University Press, 105-120.

Cooke, D., Bóna, A. and Hansen, B. [2009] Simultaneous time imaging, velocity estimation and multiple suppression using local event slopes. Geophysics, 74(6), WCA65-WCA73.

Douma, H. and de Hoop, M. [2007] Leading-order seismic imaging using curvlets. Geophysics, 72, S231-S248.

Fomel, S. [2007] Velocity-independent time-domain seismic imaging using local event slopes. Geophysics, 72(3), S139-S147.

Goldin, S.V. [1986] Seismic traveltime inversion. SEG.

Kleyn, A.H. [1977] On the migration of reflection time countour maps. Geophysical Prospecting, 25, $125-140$.

Ottolini, R. [1983] Velocity independent seismic imaging. Stanford Exploration Project, 37, 59-68.

Sword, C.H. [1987] Tomographic determination of interval velocities from reflection seismic data: the method of controlled directional reception. Ph.D. thesis, Stanfrord Exploration Project, Stanford University. 